\title{
Partial wave analysis of HADES data for two-pion produc- tion in pion-nucleon reactions
}

\author{
I. Ciepał ${ }^{1, *}$ and W. Przygoda ${ }^{2}$ \\ for the HADES Collaboration \\ A. V. Sarantsev ${ }^{3,4}$ \\ ${ }^{1}$ Institute of Nuclear Physics PAS, Kraków, Poland \\ ${ }^{2}$ Smoluchowski Institute of Physics, Jagiellonian University, Kraków, Poland \\ ${ }^{3}$ NRC "Kurchatov Institute”, PNPI, Gatchina, Russia \\ ${ }^{4}$ Helmholtz-Institute fur Strahlen- und Kernphysik, Universitat Bonn, Germany
}

\begin{abstract}
Pion beams are perfect tools to probe resonance excitations at a fixed energy, thus properties of baryonic resonances and their coupling channels can be studied. The HADES Collaboration performed a systematic scan around the second resonance region at four different pion beam momenta $0.656,0.69$, 0.748 and $0.8 \mathrm{GeV} / \mathrm{c}$ in $\pi^{-} p$ reaction. The role of the $N(1520)$ resonance in conjunction with the intermediate $\rho$-meson production has been studied in the framework of a partial wave analysis. Preliminary results on exclusive channels with one pion $\left(\pi^{-} p\right)$ and two pions $\left(n \pi^{+} \pi^{-}\right.$and $\left.p \pi^{-} \pi^{0}\right)$ in the final state are presented.
\end{abstract}

\section{Introduction}

The experimental program of the HADES Collaboration covers the measurement of dielectron emission from a compressed baryonic matter formed in heavy ion collisions and in elementary nucleon-nucleon $(n p, p p)$ and pion-proton $(\pi p)$ collisions [1]. Both objectives are complementary, the in-medium effects can be studied following the understanding of the production channels in the elementary reactions. The pion beams offer a great opportunity to reduce the unambiguities in the description of baryonic resonance excitation and their coupling to light vector mesons $\rho$ and $\omega$, which play an essential role for in-medium modifications. A systematic energy scan and high precision data are needed [2]. In 2014, a large statistics of $\pi^{-} p$ scattering were collected by HADES at four pion beam momenta $0.656,0.69,0.748$, and $0.8 \mathrm{GeV} / \mathrm{c}$ [3]. The data have been included into the multichannel partial wave analysis (PWA), developed by the Bonn-Gatchina group [4]. The extraction of cross sections in twopion final states $\left(\pi^{+} \pi^{-}\right.$and $\left.\pi^{\circ} \pi^{-}\right)$and determination of the relevant production channels have been performed. In particular, the role of resonance- $\rho \mathrm{N}$ coupling in the electromagnetic form factors of baryonic resonances are in focus of the on-going investigations [1,5].

\section{HADES detector and pion beamline}

The High Acceptance Di-Electron Spectrometer (HADES) [6], installed at GSI Helmholtzzentrum in Darmstadt, is designed to measure products of heavy ion, proton and

\footnotetext{
*e-mail: izabela.ciepal@ifj.edu.pl
} 
pion collisions up to a few $\mathrm{AGeV}$. It allows to study both hadron and rare dilepton production with the unprecedented precision. The spectrometer provides high acceptance $\left(18^{\circ}-85^{\circ}\right)$ in polar and almost full azimuthal angles, good charged particle identification $(p / K / \pi / e)$ and excellent mass resolution of 2-3\% for $e^{+} e^{-}$pairs in the light vector meson mass range.

Recently, GSI has provided a secondary pion beam to the HADES spectrometer, allowing for pion-induced collisions on proton and nuclei. A primary ${ }^{14} \mathrm{~N}$ beam is provided by the SIS18 synchrotron with an intensity of $0.8-1.0 \times 10^{11}$ ions/spill on beryllium $\left({ }^{9} \mathrm{Be}\right)$ production target, where the secondary pion beam is generated. The maximum pion intensity of $10^{6}$ pions/spill was obtained for the $0.7 \mathrm{GeV} / \mathrm{c}$ pion beam momentum with $\frac{d p}{p} \sim 2 \%$. The dedicated tracking system CERBEROS [7] allows for a reconstruction of the momentum of each pion with a resolution of $\sigma=0.3 \%$.

\section{Results}

The experiment was performed with the $\left(\mathrm{C}_{2} \mathrm{H}_{4}\right)_{n}$ (polyethylene) and pure carbon targets in order to subtract the pion- $\mathrm{C}$ reaction contribution from the data gathered with the polyethylene target.

\section{$3.1 \pi^{-} p$ elastic scattering}

The $\pi^{-} p$ elastic scattering has been reconstructed for all four pion beam momenta (for details, see [3]), and normalized in the $\theta_{\pi^{-}}^{C M}$ range of $60^{\circ}-110^{\circ}$ to the known world data (SAID database [8]), see Fig. 1, left panel. The absolute cross sections for $\pi^{-} p$ elastic scattering are presented in Fig. 1 for the two pion beam momenta, 656 (left panel) and $800 \mathrm{MeV} / \mathrm{c}$ (right panel). The error bars in Fig. 1 (HADES data, blue squares) represent systematic errors arising from the efficiency corrections, statistical errors are negligible.
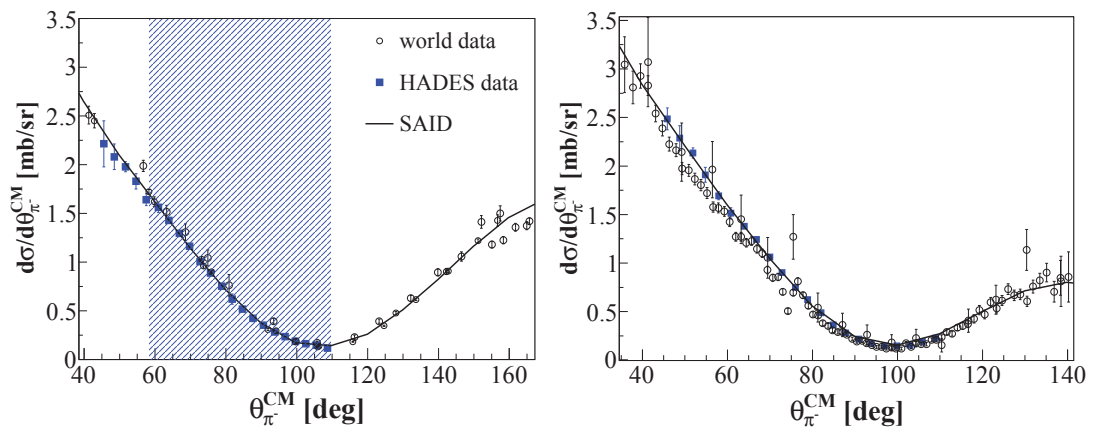

Figure 1. $\left(\pi^{-} p\right.$ elastic-scattering cross section at a beam momentum 656 (left panel) and $800 \mathrm{MeV} / \mathrm{c}$ (right panel). The HADES data (blue squares) are presented together with the world data and the current SAID solutions [8]. The marked area in the left panel refers to the region used for the normalization.

\section{$3.2 n \pi^{+} \pi^{-}$and $p \pi^{-} \pi^{0}$ final states}

Identification of $n \pi^{+} \pi^{-}$and $p \pi^{-} \pi^{0}$ in the final state allows for the kinematically complete reconstruction of the reaction channels via cuts in the missing mass distributions of the unobserved neutron or neutral pion, respectively. The $n \pi^{+} \pi^{-}$and $p \pi^{-} \pi^{0}$ reactions obtained at four 
pion beam momenta of $\mathrm{p}=0.656,0.69,0.748$ and $0.8 \mathrm{GeV} / \mathrm{c}$ corresponding to $\mathrm{W}=\sqrt{s}$ of $1.47,1.49,1.52,1.55 \mathrm{GeV}$, respectively were included in the multichannel PWA developed by the Bonn-Gatchina group $[4,9]$. In this energy range, the Crystal Ball data $[10,11]$ with the $n \pi^{0} \pi^{0}$ channel and a numerous data on pion production in photoproduction data were also obtained [12-14]. From these data, the Bonn-Gatchina PWA determines the production amplitudes and decays of the following states: $\Delta(1232) \frac{3}{2}^{+}, N(1440) \frac{1}{2}^{+}, N(1520) \frac{3}{2}^{-}, N(1535) \frac{1}{2}^{-}$, $N(1650) \frac{1}{2}^{-}$, as well as non-resonant contributions. All contributions are summed coherently. The extraction of contributions from different partial waves is performed event-by-event and based on the maximum-likelihood method. The largest contributions to the two-pion final states of interest are $\Delta(1232) \pi, N(938) \sigma$ and $N(938) \rho$. The results of the combined fit, including the HADES data, are shown in Fig. 2 for the $p \pi^{-} \pi^{0}$ invariant mass at $656 \mathrm{MeV} / \mathrm{c}$ beam momentum as an example. The systematic uncertainties of the PWA solutions are studied through the variation in the truncation at maximum orbital angular momentum of the contributing partial waves and by variations of the weights to the subsequent data sets, and the known total cross sections. The HADES data are extrapolated (acceptance corrected) based on the best PWA solution obtained.
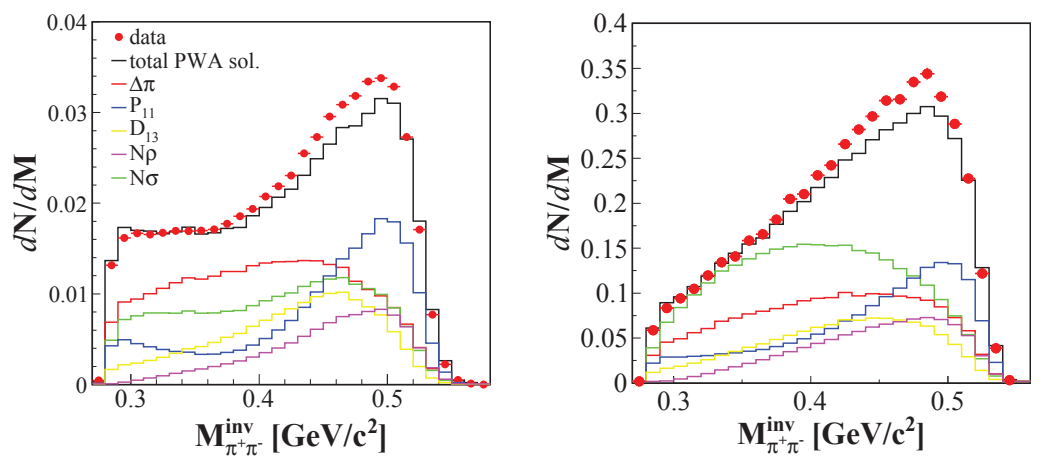

Figure 2. The acceptance corrected distributions of $\pi^{+} \pi^{-}$invariant mass at $656 \mathrm{MeV} / \mathrm{c}$ pion beam momentum (red circles) in comparison to the PWA solutions within the HADES acceptance (left panel) and after acceptance correction, deduced from the PWA solution (right panel). The lines represent PWA contributions specified in the legend. $P_{11}$ and $D_{13}$ refere to $\frac{1}{2}^{2} \frac{1}{2}^{+}$and $\frac{1}{2}^{2} \frac{3}{2}^{-}$total partial waves, respectively.

\subsection{Summary and outlook}

The HADES data greatly improve the world database of two-pion production in pion-induced experiments in the energy range around the mass of the $N(1520)$ resonance. The analysis (in progress) will allow to obtain the branching ratio of $N(1520)$ resonance in the $\rho \mathrm{N}$ decay channel and other two-pion production modes. The production of the $\rho$-meson is of utmost importance for the dielectron channel $\pi^{-} p \rightarrow n e^{+} e^{-}$, which has been measured in the same experiment [5]. The $e^{+} e^{-}$exclusive analysis allows for the direct investigation of the $N(1520)$ resonance Dalitz decay and coupling to the $\rho \mathrm{N}$ channel. Yet another information on the electromagnetic structure of the baryon transition via fit of the spin-density matrix elements can be extracted from the angular distributions of the dielectrons allowing to parametrize the differential cross sections in a model independent way. 
The pion beam program will be continued in 2019 once the SIS18 will have been upgraded to serve as an injector for the FAIR facility. The HADES setup has been equipped with large area electromagnetic calorimeter (ECAL) enabling measurements of real photons and neutral mesons via their photonic decays. The reconstruction of neutral mesons is of large importance for the complete understanding of $\pi^{-}-\mathrm{N}$ reaction dynamics and for the complete partial wave analysis of $\pi^{-} p$ reactions.

The collaboration gratefully acknowledges the support by SIP JUC Cracow (Poland), 2017/26/M/ST2/00600; TU Darmstadt (Germany), VH-NG-823; GU Frankfurt (Germany), BMBF:05P15RFFCA, HIC for FAIR, ExtreMe Matter Institute EMMI; TU München, Garching (Germany), MLL München, DFG EClust 153, BmBF 05P15WOFCA; JLU Giessen (Germany), BMBF:05P12RGGHM; NRNU MEPhI Moscow Excellence project 02.a03.21.0005, MSERF 3.3380.2017/4.; 6 IPN, IN2P3/CNRS (France); NPI CAS Rez (Czech Republic), MSMT OP VVV CZ.02.1.01/0.0/0.0/16 013/0001677, MSMT LM2015049.

\section{References}

[1] B. Ramstein, Time-Like Baryon Transitions Studies with HADES, MESON2018.

[2] W. J. Briscoe et al., Eur. Phys. J. A 51, 129 (2015)

[3] W. Przygoda, EPJ Web of Conferences 130, 01021 (2016)

[4] A. V. Sarantsev, JPS Conf. Proc. 10, 010005 (2016)

[5] F. Scozzi, EPJ Web of Conferences 130, 07021 (2016)

[6] G. Agakichiev et al. (HADES), Eur. Phys. J. A 41, 243 (2009)

[7] R. Lalik et al., 2013 IEEE Nuclear Science Symposium and Medical Imaging Conference (2013)

[8] CNS Data Analysis Center, SAID Home Page, http://gwdac.phys.gwu.edu (2018)

[9] A. Anisovich, E. Klempt, A.V. Sarantsev and U. Thoma, Eur. Phys. J. A 24, 111 (2005)

[10] S. Prakhov et al. (Crystal Ball), Phys. Rev. C 69, 045202 (2004)

[11] V. L. Kashevarov et al. ((Crystal Ball at MAMI, TAPS, and A2), Phys. Rev. C 85, 064610 (2012)

[12] J. Ahrens et al. (GDH and A2), Phys. Lett. B 624, 173 (2005)

[13] U. Thoma et al. (CB-ELSA), Phys. Lett. B 659, 87 (2008)

[14] M. Battaglieri et al. (CLAS), Phys. Rev. D 80, 072005 (2009) 